\title{
EXTRACTOR CONFIGURATIONS FOR A HEAVY ION FUSION VOLUME SOURCE*
}

\author{
O. A. Anderson ${ }^{\dagger}$, LBNL, Berkeley, CA 94720, USA
}

\begin{abstract}
In order for volume sources to deliver the current (e.g., $0.8 \mathrm{~A}$ of $\mathrm{Ar}^{+}$per module) and brightness necessary for heavy ion fusion (HIF), they must operate at high current density. Conventional extractor designs for 1 to $2 \mathrm{MeV}$ run into voltage breakdown limitations and cannot easily produce the required current rise time (about one microsecond). We discuss two systems that can overcome these volume-extraction problems. Each uses multichannel preaccelerators followed by a single channel main accelerator. Fast beam switching is done in the low energy bearmlet stages. A new design, utilizing concentric ring preaccelerators, was recently described for another application [2]. A more conventional design uses a large number of small round beamlets. In either case, the merging beamlets are angled toward the axis, a feature that dominates other focusing. By suitable adjustment of the individual angles, beam aberrations are reduced. Because of the high current density, the overall structure is compact. Emittance growth from merging of beamlets is calculated and scaling is discussed.
\end{abstract}

\section{INTRODUCTION}

For extraction of large currents with low emittance for Heavy Ion Fusion (HIF), we consider an arrangement originally proposed for a laser ion source extractor of very high perveance [2]. As shown in Figs. 1 and 2, the beamlets from a system of concentric preaccelerators are angled toward the axis. The inward momentum produces a powerful focusing effect, allowing the main $\mathrm{MeV}$ accelerator column (Fig. 1) to operate at low gradient. The angling at various radii can be adjusted to compensate for the aberrations typically present in high perveance extractors.
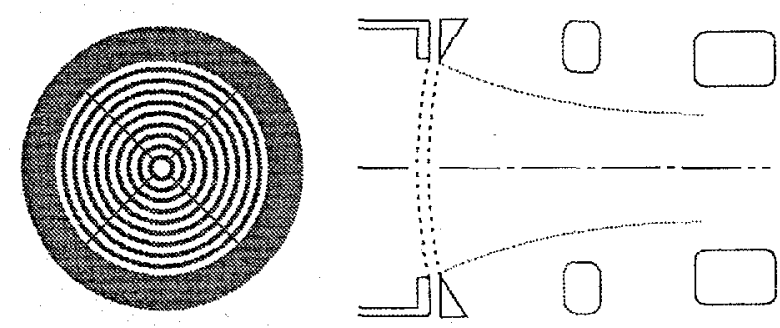

Fig. 1. Sketch of axial and cross-section views of preaccelerator grids and two-gap main extractor grids. In practice, more stages would be used in both sections.

*This work is supported by the Office of Fusion Energy Science, US DOE under contract No. DE-AC03-76SF00098.

†Email: OAAnderson@LBL.gov; also affiliated with Particle Beam Consultants, 2910 Benvenue Ave., Berkeley, CA 94705.

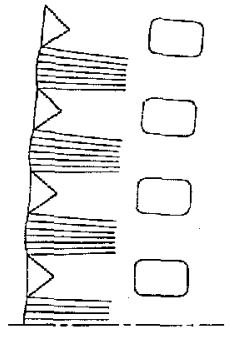

Fig. 2. Detail of preaccelerators.
The present paper studies extraction of HIF beams from conventional gas sources. The $0.75 \mathrm{~A}$ beams of $\mathrm{Ar}^{+}$are to be injected at $\sim 1.5 \mathrm{MeV}$ into an array of ESQ channels spaced about 7 $\mathrm{cm}$ in both directions. The proposed system is compact and should fit within these dimensions.

As an alternative, we also consider an older arrangement [3] using pencil beams. Figures $3 \mathrm{a}$ and $3 \mathrm{~b}$ compare axial views (before merging) for the old and new designs. In Fig. 3(a) the beamlets are arranged in idealized circular arrays to facilitate the calculations that follow.

Section 2 calculates free field energy and asymptotic emittance for both cases; it also compares transparency and brightness. Aberration control and general design considerations are discussed in section 3.
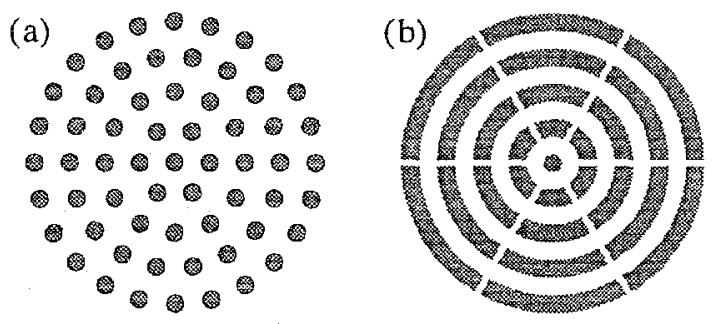

Fig. 3. Axial view of beamlets emerging from (a) beaded ring and (b) solid ring preaccelerators. Occupancy $\eta=50 \%$, number of rings $M=4$.

\section{EMITTANCE FROM MERGING}

With $X$ and $V_{x}$ the rms values of position and velocity, we define the nonrelativistic normalized rms emittance $\epsilon_{\mathrm{x}}^{2} \equiv 16 \beta^{2} \mathrm{X}^{2}\left(\mathrm{~V}_{\mathrm{x}}^{2}-\mathrm{X}^{\prime 2}\right) ; \quad \epsilon_{\mathrm{x}}=4 \beta X V_{\mathrm{x}}$ for a matched beam. Under standard conditions [4], the asymptotic emittance $\epsilon_{\mathrm{xf}}$ is then given by $\epsilon_{\mathrm{xf}}{ }^{2} \rightarrow \epsilon_{\mathrm{xi}}{ }^{2}+\epsilon_{\mathrm{xm}}{ }^{2}$ with

$$
\epsilon_{\mathrm{xm}}=\beta \mathrm{X}_{\mathrm{i}}\left(2 \mathrm{QU} \mathrm{U}_{\mathrm{n}}\right)^{1 / 2}
$$

$\epsilon_{\mathrm{xi}}$ and $\mathrm{X}_{\mathrm{i}}$ are initial quantities. The emittance due to merging $\in_{x m}$ thus adds in quadrature to the initial emittance $\epsilon_{\mathrm{xi}}=4 \beta \mathrm{X}_{\mathrm{i}} \mathrm{V}_{\mathrm{xi}}$. Q is the normalized perveance and $U_{n}$ is the initial normalized free field energy (beam shape factor) discussed in reference [4].

\subsection{Round Arrays of Round Beamlets}

In the type of array shown in Fig. 3(a), the total number $N$ of pencil beamlets is $N=3 M^{2}+3 M+1$, where $M$ is the number of beaded rings. When $\mathrm{N}$ is large [4], 


$$
\mathrm{U}_{\mathrm{n}}^{\mathrm{P}} \rightarrow \frac{4}{\mathrm{~N}}\left[\frac{3}{4}-\ln 3-\ln \eta+\frac{3}{8} \eta^{2}\right] \equiv \frac{\mathrm{f}(\eta)}{\mathrm{N}} .
$$

The superscript $\mathbf{P}$ indicates pencil beamlets. The occupancy $\eta<1$ is defined as the pencil beam diameter divided by the radial spacing. Some values of $f(\eta)$ are

\begin{tabular}{|llllll|}
\hline$\eta$ & 0.9 & 0.7 & 0.5 & 0.3 & 0.1 \\
$\mathrm{f}(\eta)$ & 0.24 & 0.77 & 1.75 & 3.56 & 7.83 \\
\hline
\end{tabular}

The approximation for $U_{n}$ is accurate to about $1 \%$ for 3 or more rings with $\eta=0.5$ but becomes less accurate as $\eta \rightarrow 1$. For $\eta=1$, unless $N$ is very large, it is better to use the tables or graphs in Ref. [4].

Exact values of $U_{n}$ as a function of the number of rings $\mathrm{M}$ are given for $\eta=0.5$ in Table 1 . These values ane represented with $1 \%$ accuracy by

$$
\mathrm{U}_{\mathrm{n}(\eta=0.5)}^{\mathrm{P}}=\frac{0.59}{\mathrm{M}^{2}(1+1.2 / \mathrm{M})}
$$

over the practical range $3 \leq \mathrm{M} \leq 12$.

\subsection{Arrays Of Concentric Tubular Beamlets}

We only have room here to discuss the case of parallel propagation where the self field $E_{s}$ is radial. For tubular beamlets, we use Gauss's law to relate $\mathrm{E}_{S}$ to $\lambda_{\mathrm{T}}(\mathrm{r})$, the line charge within radius $r$, and integrate $E_{s}{ }^{2}$. The normalized free field energy $U_{n}$ within outer radius $a$ is

$$
\mathrm{U}_{\mathrm{n}}^{\mathrm{T}}=4 \int_{0}^{\mathrm{a}} \lambda_{\mathrm{T}}^{2}(\mathrm{r}) / \lambda^{2} \mathrm{r} \mathrm{r}-1+4 \ln \left(2 \mathrm{X}_{\mathrm{i}} / \mathrm{a}\right)
$$

For the geometry of Fig. 3(b), the integrals are simplified by assuming a constant density within all tubes. (Note: the diameter of the central beamlet equals the thickness of the tubes.) We consider three values for the occupancy $\eta$ :

\begin{tabular}{|c|c|c|c|c|c|}
\hline \multicolumn{3}{|c|}{ Beaded Rings } & \multicolumn{3}{|c|}{ Solid Rings } \\
\hline $\bar{M}$ & $\begin{array}{c}\text { Un } \\
\text { eta }=1\end{array}$ & $\begin{array}{c}U_{\text {eta }}=1 / 2 \\
\text { nat }\end{array}$ & $\begin{array}{c}\text { Un } \\
\text { eta }=1\end{array}$ & $\begin{array}{c}\text { Un } \\
\text { eta }=2 / 3\end{array}$ & $\begin{array}{c}\mathrm{U} n \\
\text { eta }=1 / 2\end{array}$ \\
\hline 1 & 0.0116 & 0.2070 & 0.0 & 0.0627 & 0.0897 \\
\hline 2 & 0.0064 & 0.0882 & 0.0 & 0.0156 & 0.0289 \\
\hline 3 & 0.0034 & 0.0466 & 0.0 & 0.0070 & 0.0142 \\
\hline 4 & 0.0020 & 0.0285 & 0.0 & 0.0040 & 0.0085 \\
\hline 5 & 0.0013 & 0.0192 & 0.0 & 0.0026 & 0.0056 \\
\hline 6 & 0.0009 & 0.0138 & 0.0 & 0.0018 & 0.0040 \\
\hline 8 & 0.0005 & 0.0081 & 0.0 & 0.0011 & 0.0023 \\
\hline 10 & 0.0003 & 0.0053 & 0.0 & 0.0007 & 0.0015 \\
\hline 12 & 0.0002 & 0.0037 & 0.0 & 0.0005 & 0.0011 \\
\hline
\end{tabular}
$1 / 2,2 / 3$, and 1 with the results shown in the table.

Table 1: Free field energy comparison

For $\eta=0.5$, the ratio $U_{n}^{P} / U_{n}^{T}$ tends toward 3.5, which implies the emittance for pencil beams is larger by a factor $\sim 1.87$. Some of the values from the table for $\eta=0.5$ are plotted in Fig. 4. The useful approximation

$$
\mathrm{U}_{\mathrm{n}}^{\mathrm{T}}(\eta=0.5)=\frac{0.166}{\mathrm{M}^{2}(1+0.9 / \mathrm{M})}
$$

is accurate to $1 \%$ for $2 \leq \mathrm{M} \leq 40$.

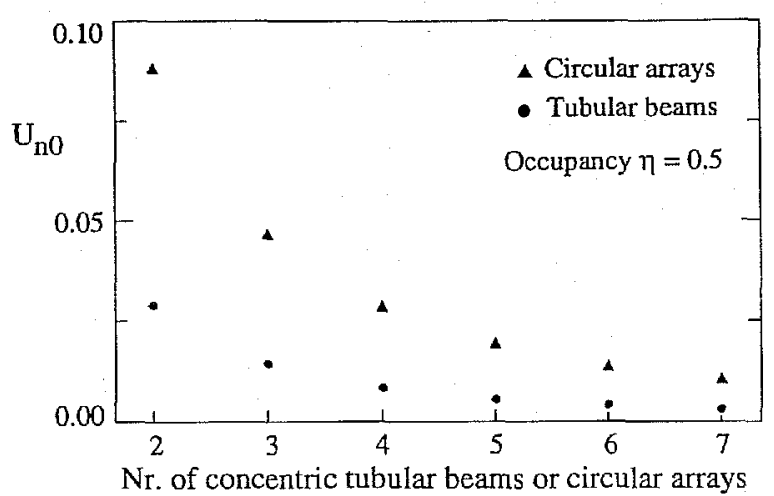

Fig. 4. Normalized free field energy $U_{h}$ for the two configurations of Fig. 2.

\subsection{RMS Beam Size}

The maximum radius a $a_{\max }$ is defined as the largest radius in Fig. 3 (a) or (b). The rms size $X_{i}$ as a function of $a_{\max }$, occupancy $\eta$, and number of rings $M$ is easily calculated for beadel rings; see Ref. [4]. For solid rings, we have evaluated $X_{i}$ for the cases $\eta=1 / 2(5 a)$ and $\eta=2 / 3(5 b)$ :

$$
\begin{aligned}
& \frac{4 X_{i}^{2}}{a^{2}}=1+\frac{2}{m}-\frac{3}{m^{2}}+\frac{2}{m^{2}\left(m^{2}+2 m-1\right)} ; m=4 M+1 \\
& \frac{4 X_{i}^{2}}{a^{2}}=1+\frac{1}{\mu}-\frac{1.5}{\mu^{2}}+\frac{3}{\mu^{2}\left(\mu^{2}+\mu-0.5\right)} ; \mu=3 M+1 .
\end{aligned}
$$

For the case $M=4$ illustrated in Fig. 3, the values are $\mathrm{X}_{\mathrm{i}}(\eta=1 / 2)=0.5 \mathrm{a}_{\max }(1+0.05)$ and $\mathrm{X}_{\mathrm{i}}(\eta=2 / 3)=$ $0.5 \mathrm{a}_{\max }(1+0.03)$. Of course, $X_{i}(\eta=1)=0.5 \mathrm{a}_{\max }$, the usual value for a uniform beam. The corrections are just a few percent and may be neglected for purposes of estimation. For beaded rings, as well, it usually suffices to write $\mathrm{X}_{\mathrm{i}} \approx 0.5 \mathrm{a}_{\max }$.

\subsection{Merge Emittance}

Equation (1) with (3) and (4) gives approximately

$$
\begin{aligned}
& \epsilon_{x m(\eta=0.5)}^{\mathrm{p}} \approx \frac{\mathrm{a}_{\max }}{\mathrm{M}} \frac{\beta}{\sqrt{ } 2}\left[\frac{0.59 \mathrm{Q}}{1+\varepsilon_{\mathrm{p}}}\right]^{1 / 2} \\
& \epsilon_{\mathrm{xm}(\eta=0.5)}^{\mathrm{T}} \approx \frac{\mathrm{a}_{\max }}{\mathrm{M}} \frac{\beta}{\sqrt{2}}\left[\frac{0.166 \mathrm{Q}}{1+\varepsilon_{\mathrm{T}}}\right]^{1 / 2}
\end{aligned}
$$

with correction terms $\varepsilon_{\mathrm{p}}=1.2 / \mathrm{M}$ and $\varepsilon_{\mathrm{T}}=0.9 / \mathrm{M}$. For constant current $I, Q \sim I / \beta^{3}$ and $\in_{m}-\beta^{-1 / 2} \sim V^{-1 / 4}$. Omitting $\varepsilon_{p}, \varepsilon_{T}$ and the corrections to $X_{i}$ in Eq. (5) gives the rough scaling for large $M$ (SI units, $€$ in $\pi$-m-rad):

$$
\begin{gathered}
\epsilon_{x m(\eta=0.5)}^{P} \approx C \frac{Z^{1 / 4}}{A^{1 / 4}} \frac{a_{\max }}{M} \frac{I^{1 / 2}}{V^{1 / 4}} \\
\epsilon_{x m(\eta=0.5)}^{T} \approx 0.53 C \frac{Z^{1 / 4}}{A^{1 / 4}} \frac{a_{\max }}{M} \frac{I^{1 / 2}}{V^{1 / 4}}
\end{gathered}
$$

with $Z$ and $A$ the ion charge and mass numbers. The constant $C=0.02$ with $\eta=0.5$. For other occupancies, $\epsilon_{\mathrm{xm}}^{\mathrm{P}}$ can be adjusted using Eq. (2), the table below it, or 


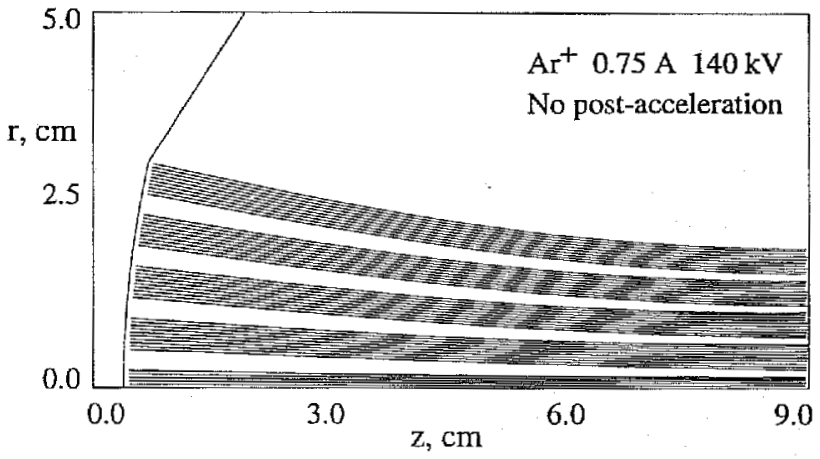

Fig. 5. Beam simulation to check emittance growth from free field energy in merging beams.

the information in Ref. [4]. For $\epsilon_{\mathrm{xm}}^{\mathrm{T}}$, one can use Table 1 which, incidentally, indicates that the ratio $\in_{\mathrm{xm}}^{\mathrm{P}} / \in_{\mathrm{xm}} \mathrm{T}$ becomes even larger than 3.5 as $\eta$ increases.

\subsection{Checking Against Particle Codes}

Using WARPxy, the normalized emittance growth term $\epsilon_{\mathrm{m}}^{\mathrm{P}}$ for the merging pencil beam case was simulated for uniform transport using parameters representative of injection into an HIF ESQ channel [5]. The result, $0.4 \pi$-mm$\mathrm{mr}$, agreed well with the prediction of Eq. (6a). A typical initial thermal emittance $\epsilon_{\mathrm{xi}}$ of $0.3 \pi-\mathrm{mm}$-mr would then give a combined value $\epsilon_{\mathrm{xf}}$ of $0.5 \pi$-mm-mr.

Using a different code [6], tubular beams were simulated as shown in Fig. 5. This case, somewhat different from that analyzed above, studied radial compression in drifting beams; $\epsilon_{\mathrm{xm}}^{\mathrm{T}}$ was predicted by (6b) to be roughly $0.6 \pi$ mm-mr. Fig. 6 shows the exit emittance diagram at the waist. The value 0.41 for emittance is reasonable since the beamlets are not yet completely merged at this point.

\subsection{Other Differences}

Pencil beams and tubular beams differ in other ways than emittance growth. The extraction hardware may be easier to fabricate in the first case. But a tubular beam design has the advantage that the essentially 1-D radial geometry precludes non-linear field distortion from neighboring

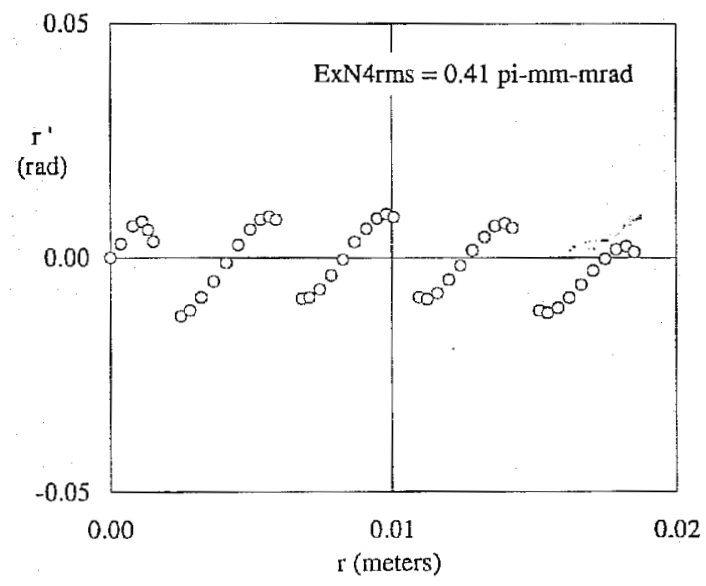

Fig. 6. Emittance phase plot at exit of Fig. 5 . beamlets that may occur with pencil beams before merging begins. Also, for a given occupancy $\eta$, the transparency for tubular beams is higher by the factor $4 / \pi \eta$, increasing the brightness for cases of fixed current density.

\section{EXTRACTOR SYSTEMS}

In some high perveance designs for HIF, the high-voltage portion may produce beam aberrations. In the two-stage system of Figs. 1 and 2 the preaccelerator beamlets can be steered to reduce these aberrations. Figure 7 shows phase plots at the exit for a laser plasma extractor design with $40 \mathrm{~A}$ of $\mathrm{Xe}^{+8}$. The main extractor gap was smaller than the diameter of the preaccelerator array, producing the overfocusing of outer beamlets seen on the left side of Fig. 7. Beam steering removed most of the aberrations.
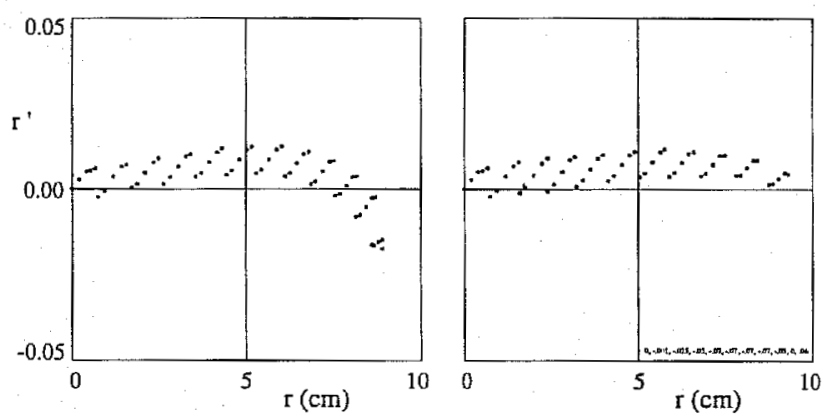

Fig. 7. Aberration control by pre-steering of beamlets: uncorrected (left), corrected (right).

The preaccelerators and the main accelerator channel for extraction from a conventional $\mathrm{Ar}^{+}$source have been studied separately. Using the above analysis, we find that the number of rings $M$ required to produce an acceptable emittance is not so large that the individual units become impracticably small. The ring-to-ring spacing will be typically $\sim 0.7 \mathrm{~cm}$ so that construction should not be difficult. The electric field for the preaccelerator cases studied is well under $100 \mathrm{kV} / \mathrm{cm}$.

The length of the main column is controlled by the curvature of the preaccelerator exit array seen in Fig. 1. Preliminary simulations show that it should be possible to accelerate the beam to $1.5 \mathrm{MeV}$ and compress it to the required diameter while maintaining an acceptable field gradient. Further studies are in progress.

\section{REFERENCES}

[1] E.P. Lee, et al., Fus. Eng. Des. 32-33, 323 (1996).

[2] O.A. Anderson and B. Grant Logan, "Low Emittance Beam Extraction from Laser-Driven Multicharged Ion Sources," Proc. 6th EPAC Conf., Stockholm, 1998.

[3] O.A. Anderson, proceedings of US-Japan Workshop on Negative Ion Beams, JAERI, Japan, Nov. 14-17, 1988.

[4] O.A. Anderson, "Emittance Growth from Merging Arrays of Round Beamlets," Fusion Eng. and Design 32-33, 209-217 (1996).

[5] David P. Grote, personal communication, LBNL, March 1999. The parameters were provided by J. W. Kwan.

[6] S. Humphries, J. Comput. Phys. 125, 488 (1996). 
Subject: Re: Request for LBNL Report Number

Date: Mon, 26 Jul 1999 16:53:06-0700

From: "Anne S. Fleming" <astleming@1bl.gov>

Organization: Lawrence Berkeley National Laboratory

References: 1

To: Lynn Heimbucher $\leq$ LCHeimbucher@ lbl.gov>

LBNL-42441 was requested by Sarah st. Hill, but never received. OK?

Netscape commerce server wrote:

$>$ LCHeimbucheralbI.gov (Lym Heimbucher) sent the following request:

$>$

> Original Title: Time Dependent Beam Focusing at the DARHT-II Injector Diode

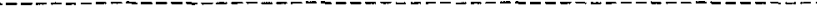

$>$ START OF RECORD

$>$ AUTHOR=EYION, $S$.

$>$ DOC TITLE=Time dependent beam focusing at the darht-ii injector diode

$>$ DIVISION ID =AFRD

$>$ DOC NUM TYPE $=\angle B N L$

$>$ DOC_FORMAT $=$ UNKNOWN

$>$ ACCESS_LEVEI $=0$

$>$ CONTACT_ID $=801534$

$>$ CONTACT=Linn Heimbucher

$>$ CONTACT_ADDR $=47-112$

$>$ CONTACT_PHONE $=(510) 486-6372$

$>$ CONTACT_EMAIL $=L C H e i m b u c h e r e I b I$.gov

$>$ END OF RECORD

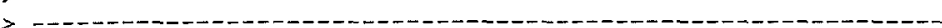

Fleming, Anne S. <asfleming@1bl.gov>

Wer Completerar

Report Coordinator

Lawrence Berkeley National Laboratory

Library, TED

Adofo Address Book 


\section{Thank you!}

Your request has been sent to Report Coordination.

Report Coordination will send back to you the unique report number that bas been assigned to your request.

After you receive your unique report number, you may submit your document to Report Coordination along with a completed document submission form.

Below is a copy of your request:

Request submitted: Jul 26, 1999

AUTHOR Eylon, S.

TITLE Time D

DIVISION_ID AFRD

DOC_FORMAT UNKNOWN

DOC_NUM_TYPE LBNL

CONTACT_ID 801534

CONTACT Lynn Heimbucher

CONTACT_ADDR 47-112

CONTACT_PHONE (510)486-6372

CONTACT_EMAIL LCHeimbucher@lbl.gov

Return to our Home Page 